\title{
Case - Ex-vivo aneurysm resection, autotransplantation, and aorto-renal bypass in a solitary kidney with fibromuscular dysplasia
}

Gautamn Sarwal, MD MEd(cand) ${ }^{1}$; Hilary L. Brotherhood, $\mathrm{MD}^{2}$, Edmund C.P. Chedgy, MBBS, MSc, FRCS (Urol) ${ }^{3}$; David C. Taylor, MD, FRCPC ${ }^{4}$; Alex G. Kavanagh, MD, MPH, FRCPC ${ }^{3}$ ${ }^{1}$ PGY-3 Vascular Surgery, University of British Columbia; ${ }^{2}$ PGY-5 Urological Surgery, University of British Columbia; ${ }^{3}$ Department of Urological Sciences, Vancouver General Hospital; ${ }^{4}$ Division of Vascular Surgery, Vancouver General Hospital; Vancouver, BC, Canada

Cite as: Can Urol Assoc J 2018 May 14; Epub ahead of print. http://dx.doi.org/10.5489/cuaj.5133

Published online May 14, 2018

$* * *$

\section{Introduction}

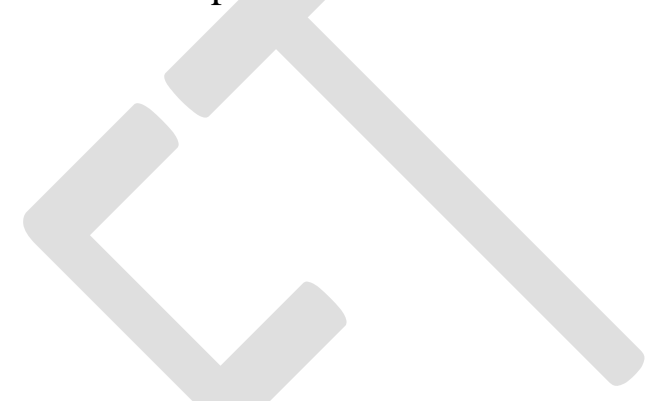

Renal artery aneurysms are rare, with an incidence of $0.1 \% .^{1-3}$ Their clinic-pathological features and anatomical classification is outlined in Table 1. RAAs are typically unilateral, solitary, saccular, and right-sided, measuring on average $1.3-3.8 \mathrm{~cm} .{ }^{3,4}$ In upto $30 \%$ of cases, multiple aneurysms are found and 10-20\% present bilaterally. ${ }^{3,4}$ Controversy over the criteria for selecting endovascular vs. open repair persists, with ex-vivo resection and renal autotransplantation being described for complex distal RAAs in case reports. ${ }^{4-6}$

Fibromuscular dysplasia (FMD) is a non-inflammatory, non-atherosclerotic disease affecting arterial walls causing stenosis, or aneurysmal degeneration. ${ }^{6}$ Renal artery involvement is most common and 5-10\% of patients with FMD present with RAAs. ${ }^{6}$

We present our multidisciplinary approach to a 21-year-old male presenting with multiple complex distal RAAs in a solitary kidney with FMD requiring ex-vivo aneurysm resection, autotransplantation , and an aorto-renal bypass. The case described here is particularly unique, given the patient's young age and solitary kidney, the loss of which would have resulted in lifelong hemodialysis. Additionally, this patient was previously presented in the literature by Smart et al (2010) after collapsing during hockey practice with a ruptured left renal artery aneurysm that necessitated a nephrectomy. ${ }^{7}$ Magnetic resonance arteriography (MRA) following nephrectomy did not demonstrate any evidence of contralateral aneurysm formation. ${ }^{7}$ 


\section{Case report}

A 21-year-old male, with a history of left nephrectomy secondary to a ruptured RAA at age 10, presented with atraumatic right flank pain. Cross-sectional imaging demonstrated three complex distal RAAs, the largest measuring 5.6 x 4.4 x 3.6cm with the smaller aneurysms measuring 1.4 $\mathrm{x} 1.6 \mathrm{~cm}$ and $2.5 \mathrm{x} 2.2 \mathrm{~cm}$ respectively.

A pre-operative MRA (figure 1) demonstrated a complex cluster of hilar aneurysms and smaller aneurysms involving posterior branches of the IIA and profunda femoris bilaterally. A computed tomography arteriogram (CTA) arch to vertex was negative for aneurysm.

A renal arteriogram (figure 1) demonstrated a proximal superior and inferior branch arising from the right main renal artery with all three aneurysms originating from the superior branch. DynaCT revealed the majority of the renal parenchyma was supplied by this superior branch. Given multiple aneurysms, there was concern for a connective tissue disorder or fibromuscular dysplasia.

In view of aneurysm size, complexity, and a solitary kidney, an interdisciplinary approach with vascular surgery was chosen due to the risk of rupture and potential implication for lifelong renal replacement therapy. A decision was made to proceed with ex-vivo aneurysm resection, autotransplantation and a concomitant aortorenal bypass to replace the diseased renal artery and prevent future aneurysmal degeneration. The patient's preoperative creatinine was 93 $\mu \mathrm{mol} / \mathrm{L}$.

Via a midline laparotomy, the right hemicolon was mobilized medially and duodenum kocherized in order to expose the right kidney, inferior vena cava (IVC) and perirenal aorta. The right main renal artery was isolated, double ligated and divided followed by the right renal vein. A small renal vein stump was left on the IVC to facilitate re-anastomosis. Once complete, the kidney, with the ureter still attached, was explanted (figure 2). It was subsequently placed in cold saline $\left(4^{0} \mathrm{C}\right)$ for cooling and flushed with University of Wisconsin (UW) preservative solution. The aneurysms were examined revealing three outflow vessels off the largest aneurysm and two off the smaller ones. These were then excised and sent to histopathology. Histological examination of the resected aneurysms and artery showed marked intimal thickening and mural fibrosis with obliteration of the tunica media consistent with medial type FMD.

Great saphenous vein (GSV) was harvested and used as conduit for revascularizing the kidney with a distal aortorenal bypass (figure 3). Three short segments of GSV were used to reconstruct the outflows from the resected aneurysms. These three segments were spliced to create a common orifice, and the upper and lower pole arteries were joined creating a five-fold outflow vessel. This was spliced and anastomosed end-to-end with reversed GSV to the aorta for bypass. In total, five branches were reconstructed including one upper pole, one lower pole and three renal aneurysm branches. The renal vein was then re-anastomosed to the IVC using 4-0 prolene in a continuous running fashion. After releasing all clamps, the kidney appeared wellperfused with urine output in 20 minutes. There were no intraoperative complications. The total clamp time to the kidney was three hours and ten minutes. Creatinine immediately post- 
operatively increased to $144 \mu \mathrm{mol} / \mathrm{L}$ but returned to baseline at the time of discharge a week later.

A three-month follow-up ultrasound demonstrated excellent arterial hemodynamics with no renal abnormalities. The patient remained normotensive with renal function at baseline.

\section{Discussion}

In the literature, the indications and modality of RAA repair remains debated. Endovascular approaches are increasingly applied with proximal aneurysms, but open resection is often required for distal RAAs. Currently accepted indications for RAA intervention include size $\geq$ $2 \mathrm{~cm}$, females of childbearing age, symptomatic aneurysms including rupture, hematuria and refractory hypertension. ${ }^{1,3}$ Open resection and reconstruction options include aneurysmectomy with primary repair or patch angioplasty, interposition grafting, bypass, or ex-vivo resection with autotransplantation as described in case reports. ${ }^{1,3}$ Indications for $e x$-vivo resection include ruptured RAAs and patients with complex distal RAAs with the need for suturing adjacent to the renal parenchyma. To protect against ischemia in such cases, topical cooling and perfusion of the kidney with a preservative solution has been shown to be beneficial. ${ }^{10}$

With endovascular advances, covered stenting is used to exclude main renal artery aneurysms with aneurysm coiling and embolization reserved for distal and parenchymal aneurysms. ${ }^{1,3}$ Historically, patients with complex distal RAAs underwent nephrectomy, ${ }^{3}$ however, given this patient's young age and solitary kidney, this would have implied lifelong renal replacement therapy. Endovascular coil embolization was not feasible either given the risk of occluding the superior branch that was shown on DynaCT as perfusing the majority of this patient's solitary kidney. Such complex aneurysms have been treated with ex-vivo resection with safety and durability as described in case reports, even in solitary kidneys. ${ }^{4-6}$ Accordingly, we elected to proceed with a multidisciplinary approach to treat the diseased artery and its aneurysms.

We chose to resect the entire main renal artery and proceed with an adjunctive aortorenal bypass to prevent future aneurysmal degeneration in the presumed diseased artery.

Reconstructing distal segmental branches post aneurysm resection remained time-consuming but preservation of the solitary kidney was critical in this patient. Aortorenal bypass has been described as a potential technique in treating main renal artery aneurysms however never in conjunction with ex-vivo aneurysm resection.

Larger case series have examined the short and long-term results and durability of ex-vivo resection and autotransplantation. For example, Morin et al (2012) described a series of nine patients with solitary kidneys undergoing ex-vivo resection and found no detrimental effect on renal function 90 months post in follow-up. ${ }^{11}$ Recently, Laser et al (2015) described their series of 14 hilar RAAs managed by ex-vivo resection reporting no perioperative mortality, stable renal function and vascular patency 19-months post in follow-up. ${ }^{12}$ The largest series of ex-vivo aneurysm resection and autotransplantation evaluated 67 procedures with a nine year follow-up reporting no perioperative mortality, freedom from hypertension in $43 \%$ of patients, and a 
patency of $88 \%$ after nine years. ${ }^{13}$ They concluded that ex-vivo aneurysm resection eliminates the risk of aneurysm rupture, treats hypertension, preserves renal function and confers long-term vascular patency. ${ }^{13}$ However, to the best of our knowledge, there is no published case or longterm results involving a complex distal RAA in a solitary kidney with FMD that was treated with ex-vivo aneurysm resection, autotransplantation and an aortorenal bypass to reconstruct the diseased renal artery.

In conclusion, ex-vivo aneurysm resection and autotransplantation with bypass is a safe and feasible procedure for treating complex distal RAAs. The long-term data on this technique with solitary kidneys remains isolated to a few case reports. Further laboratory examination and imaging is necessary at evaluating the optimal follow-up duration given this patient's previous aneurysm history, the impact of ex-vivo ischemia and the durability of this hybrid urology and vascular approach to treating complex distal RAAs and the diseased renal artery.

\section{Learning points}

- RAAs are typically unilateral, saccular, solitary and favor the right side, measuring on average 1.3 to $3.8 \mathrm{~cm}$

- Indications for intervention include size $\geq 2 \mathrm{~cm}$, females within childbearing age, symptomatic aneurysms including rupture, and refractory hypertension

- Indications for ex vivo resection and autotransplantation may include ruptured RAAs and patients with complex distal RAAs;

- The unpredictable complexity of hilar RAAs may result in some lesions necessitating a partial or complete nephrectomy 


\section{References}

1. Gonzalez J, Esteban M, Linares AE, Martinez-Salamanca JI. Renal artery aneurysms. Curr Urol Rep 2014; 15:376.

2. Hermiz SJ, Afshari A, Smith RS, et al. Ruptured renal artery aneurysm presenting as acute abdominal pain. Amer Surg 2016; 82:E67-68.

3. Coleman DM, Stanley JC. Renal artery aneurysms. J Vasc Surg 2015; 62:779-85.

4. Knoblock K, Wiebe K, Lichtenberg A, et al. Ex-vivo repair and renal autotransplantation for complex renal artery aneurysms in a solitary kidney. Ann Vasc Surg 2005; 19:1-4.

5. Berloco PB, Sandri GBL, Guglielmo N, et al. Bilateral ex-vivo repair and kidney autotransplantation for complex renal artery aneurysms: a case report and literature review. Int J Urol 2014; 21:219-21.

6. Palcau L, Gouicem D, Joguet E, et al. Solitary kidney with renal artery aneurysm repaired by ex-vivo reconstruction. Vasc Endovasv Surg 2014; 48(5-6):430-33.

7. Smart GW, Crowley JS, Lavoie DJ. Collapse of a hockey player: ruptured aneurysm of the renal artery. CMAJ 2010; 182(8):803.

8. Wayne EJ, Edwards MS, Stafford JM, et al. Anatomic characteristics and natural history of renal artery aneurysms during longitudinal imaging surveillance. J Vasc Surg 2014; 60:448-52.

9. Rubdback JH, Rizvi A, Rozenblit GN, et al. Percutaneous stent management of renal artery aneurysms: updated classification of RAAs with management implications. $J$ Vasc Interv Radiol 2000; 11:1189-93.

10. Pfeiffer T, Reiher L, Grabitz K, et al. Reconstruction for renal artery aneurysm: operative techniques and long-term results. J Vasc Surg 2003; 37:293-300.

11. Morin J, Chavent B, Duprey A, et al. Early and late results of ex-vivo repair and autotransplantation in solitary kidneys. Eur J Vasc Endovasc Surg 2012; 43:716-20.

12. Laser A, Flinn WR, Benjamin ME. Ex-vivo repair of renal artery aneurysms. J Vasc Surg 2015; 62:606-9.

13. Duprey A, Chavent B, Meyer-Bisch V, et al. Ex-vivo renal artery repair with kidney autotransplantation for renal branch artery aneurysms: long-term results of sixty-seven procedures. J Vasc Endovasv Surg 2016; 51:872-9. 
Figures and Tables

\section{Table 1. Pathology of renal artery aneurysms}

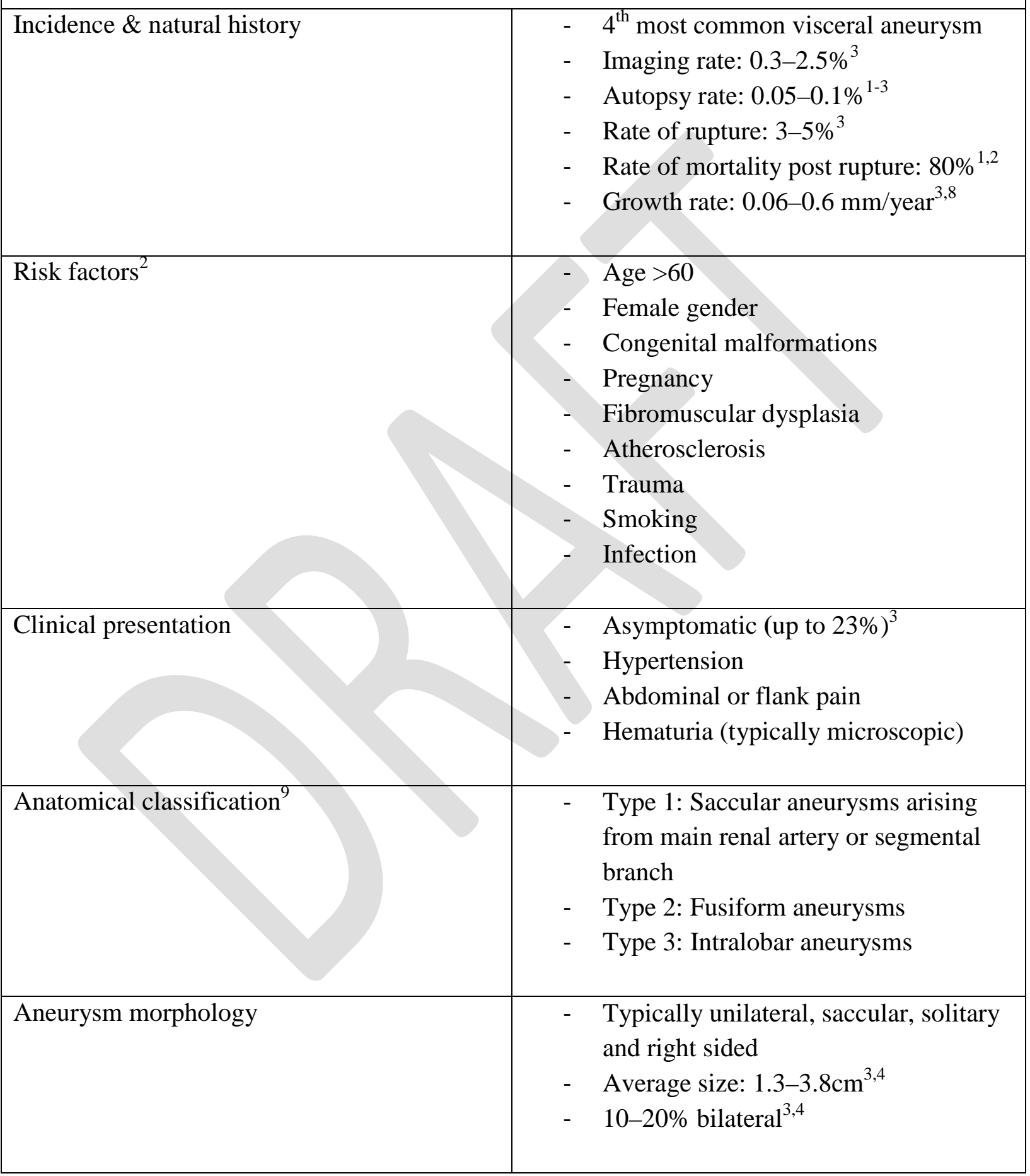

